\title{
Review Article \\ Neurotoxicity Caused by the Treatment with Platinum Analogues
}

\author{
Sousana Amptoulach and Nicolas Tsavaris \\ Oncology Unit, Department of Pathophysiology, Laikon General Hospital, Athens University School of Medicine, 11527 Athens, Greece \\ Correspondence should be addressed to Nicolas Tsavaris, tsavari1@otenet.gr
}

Received 10 September 2010; Revised 29 March 2011; Accepted 4 May 2011

Academic Editor: Athanassios Tsakris

Copyright ( 12011 S. Amptoulach and N. Tsavaris. This is an open access article distributed under the Creative Commons Attribution License, which permits unrestricted use, distribution, and reproduction in any medium, provided the original work is properly cited.

\begin{abstract}
Platinum agents (cisplatin, carboplatin, and oxaliplatin) are a class of chemotherapy agents that have a broad spectrum of activity against several solid tumors. Toxicity to the peripheral nervous system is the major dose-limiting toxicity of at least some of the platinum drugs of clinical interest. Among the platinum compounds in clinical use, cisplatin is the most neurotoxic, inducing mainly sensory neuropathy of the upper and lower extremities. Carboplatin is generally considered to be less neurotoxic than cisplatin, but it is associated with a higher risk of neurological dysfunction if administered at high dose or in combination with agents considered to be neurotoxic. Oxaliplatin induces two types of peripheral neuropathy, acute and chronic. The incidence of oxaliplatin-induced neuropathy is related to various risk factors such as treatment schedule, cumulative dose, and time of infusion. To date, several neuroprotective agents including thiol compounds, vitamin E, various anticonvulsants, calcium-magnesium infusions, and other nonpharmacological strategies have been tested for their ability to prevent platinum-induced neurotoxicity with controversial results. Further studies on the prevention and treatment of neurotoxicity of platinum analogues are warranted.
\end{abstract}

\section{Introduction}

Platinum drugs are among the most important cytotoxic drugs available to oncologists. Although they share some structural similarities, there are also marked differences in their therapeutic use, pharmacokinetics, and adverse effect profiles [1-3]. Cisplatin is the first agent of platinum drugs, which was approved in 1978 for the treatment of testicular and ovarian cancer [4]. In view of its considerable toxicity profile, many attempts have been made to develop analogues with less toxicity, increased efficacy, or both. Carboplatin is a second-generation platinum drug with equivalent activity, in some cancer types, to cisplatin. Carboplatin is often administered in combination with a taxane as a first-line treatment for ovarian cancer $[5,6]$. Lung cancer is also treated with carboplatin in combination with vinorelbine, gemcitabine, or paclitaxel [7]. Oxaliplatin, a third-generation platinum drug, is the standard of treatment, together with 5-fluorouracil/leucovorin ( $5 \mathrm{FU} / \mathrm{LV}$ ), for locally advanced and metastatic cancer of the colon and rectum [8]. This paper aims to highlight the neurotoxicity of commonly used platinum agents and published data on certain compounds that have been reported to have protective effects when administered simultaneously with these agents.

\section{Cisplatin}

Peripheral neurotoxicity is the most important dose-limiting problem associated with cisplatin [9]. A number of pathophysiological mechanisms have been proposed to explain this phenomenon, with some data suggesting that cisplatin kills malignant cells and peripheral neurons by means of a similar mechanism of apoptosis [10]. Peripheral neurotoxicity develops in approximately $50 \%$ of patients receiving cisplatin [11], but the onset of toxicity is delayed until a cumulative dose higher than $300 \mathrm{mg} / \mathrm{m}^{2}$ has been given $[12,13]$. Signs and symptoms of peripheral neurotoxicity involve the upper and lower extremities and include loss of vibration sense, loss of position sense, tingling paraesthesia, weakness, tremor, and loss of taste [14-16]. Seizures and leukoencephalopathy have also been described [17, 18]. After discontinuation of treatment, the neurological dysfunction may gradually improve, but it may persist for a period of time, or it can be permanent $[11,19]$. 
Cisplatin is ototoxic. Tinnitus and hearing loss have been observed in up to $31 \%$ of patients treated with initial intravenous cisplatin dose of $50 \mathrm{mg} / \mathrm{m}^{2}[20,21]$. Transient hearing loss and mild audiometric abnormalities were observed in $30 \%$ of patients receiving $150 \mathrm{mg} / \mathrm{m}^{2}$ of cisplatin $[22,23]$. The mechanisms of cisplatin-induced damage to the outer hairy cells of the cochlea probably include the formation of reactive oxygen radicals and depletion of glutathione [24]. Other risk factors include simultaneous use of other potentially ototoxic agents (e.g., aminoglycosides), previous cranial irradiation, preexisting renal dysfunction, or inner ear damage [21, 23, 25, 26].

\section{Carboplatin}

Carboplatin is considered to be less neurotoxic than cisplatin [27]. Neurological dysfunction is a side effect in the carboplatin-based regimen but appears later on and mostly after the administration of carboplatin at high-dose levels or in combination with other cytotoxic agents known to be neurotoxic (e.g., taxanes) $[5,6,28]$. Only $4-6 \%$ of patients who receive carboplatin may develop peripheral neuropathy [29]. Pretreated patients with other neurotoxic agents (e.g., cisplatin, and etoposide) and those who are older than 65 years have a higher risk [29, 30].

Ototoxicity, after therapy with carboplatin, is thought to be rare. A small proportion of patients, about 1.1\%, show symptoms such as tinnitus or subclinical audiographical changes [31].

\section{Oxaliplatin}

Oxaliplatin is more like cisplatin in its potential to produce significant neurological dysfunction (Table 1). Peripheral neuropathy is the most common dose-limiting toxicity of oxaliplatin, and it is one of the major causes of discontinuation of therapy. Sensory peripheral neuropathy caused by administration of oxaliplatin is distinguished in 2 forms: (1) an acute peripheral sensory neuropathy that may appear during the administration of the drug or after the first few drug infusions and (2) a chronic dose-limiting cumulative peripheral sensory neuropathy. The mechanisms underlying these two forms of oxaliplatin-induced peripheral neuropathy have not been clearly defined. Acute neurotoxic effects may result from the impairment of voltage-gated sodium channels and occur approximately in $85-95 \%$ of all patients exposed to oxaliplatin [32, 33]. Symptoms consist mainly of paresthesias and dysesthesias in the extremities and the perioral region and are exacerbated by cold exposure.

One other very rare manifestation of acute oxaliplatininduced neurotoxicity is laryngopharyngeal dysesthesia, a transient sensation of difficulty in breathing without evidence of respiratory distress $[20,34]$. This transient syndrome affects approximately $1-2 \%$ of patients and recovers between cycles [35]. The risk of acute neuropathy appears to be lower if oxaliplatin is administrated in a dose of $85 \mathrm{mg} / \mathrm{m}^{2}$ every 2 weeks rather than $130 \mathrm{mg} / \mathrm{m}^{2}$ every 3 weeks $[33,34]$.

The most accepted mechanism of the chronic form oxaliplatin-induced neurotoxicity is decreased cellular metabolism and axonplasmatic transport resulting from the accumulation of oxaliplatin in the dorsal root ganglia cells. As a result, oxaliplatin produces symmetrical, axonal, and sensory distal neuropathy [36]. Neurological symptoms in this form of sensory neurotoxicity are dominated by pronounced paresthesias and dysesthesias of the extremities and dysfunction of fine sensory-motor coordination which may result in impairment of daily life [20,37]. Other rare atypical neurosensory symptoms associated with higher cumulative dose of oxaliplatin (higher than $1000 \mathrm{mg}$ ) are those of spinal cord compression (Lhermitte sign) and urinary retention [38]. The incidence of chronic oxaliplatininduced peripheral neuropathy is related to various risk factors such as cumulative dose, treatment schedule, and time of perfusion [39]. The peripheral sensory neuropathy induced by oxaliplatin tends to improve after treatment is stopped. Symptoms are partly reversible in approximately $80 \%$ of patients and resolve completely in about $40 \%$ of patients 6-8 months after the discontinuation of oxaliplatin treatment $[36,40]$.

Ototoxicity due to administration of oxaliplatin is very uncommon [41, 42].

\section{Prevention and Treatment of Platinum-Induced Neurotoxicity}

Many studies have examined the efficacy of a number of potential neuroprotective agents administered together with platinum analogues. The use of these agents generally aims to reduce the incidence and severity of the neurotoxicity without impairing the antitumor efficacy of the platinum drugs.

5.1. Thiol Compounds. Three thiol compounds have been studied as neuroprotective agents in patients receiving cisplatin: amifostine, glutathione, and the melanocortin Org 2766.

Among these agents, glutathione seems to have some neuroprotective effects in platinum-induced neurotoxicity. Glutathione has been studied as a chemoprotective agent in patients receiving chemotherapy with cisplatin in small randomized trials. Published data are conflicting as some of the studies have shown that glutathione may provide neuroprotection in patients treated with cisplatin without altering its antineoplastic effect [37, 43, 44], while others found no reduction in toxicity $[45,46]$. Cascinu et al. were the first to study the potentially protective effect of glutathione on oxaliplatin neurotoxicity in a randomized, placebo-controlled clinical trial [47]. This study showed that glutathione can exert a beneficial effect on oxaliplatininduced neurotoxicity without interferences with oxaliplatin antitumor activity. Other studies, however, have shown no benefit from the use of glutathione for preventing the oxaliplatin-induced peripheral neuropathy [48]. In addition, the fact that elevated intracellular levels of glutathione have been correlated with increased resistance to platinum agents raises some concerns over the use of glutathione as a neuroprotective agent [48-50]. More trials are needed 
TABLE 1

\begin{tabular}{lll}
\hline Comparison of cisplatin- and oxaliplatin-induced neurotoxicity & Cisplatin & Oxaliplatin \\
\hline Dose limiting toxicity & Peripheral neurotoxicity & Peripheral neurotoxicity \\
Symptoms & Paresthesia & Paresthesia, sensory ataxia, and dysesthesia \\
Location & Extremities & Extremities, perioral area \\
Time-course onset & Delayed & Acute and delayed \\
After treatment & deterioration & Recovery \\
Accompanying toxicities & Ototoxicity & Laryngospasms \\
Precipitating factors & None & Exposure to cold \\
\hline
\end{tabular}

to confirm the safety and usefulness of glutathione as a protective agent against platinum-induced neurotoxicity.

In respect to cisplatin-induced ototoxicity, there is some evidence that amifostine may be useful as a protective agent $[20,51]$.

5.2. Vitamin E. The neuroprotective role of vitamin $E$ against cisplatin neurotoxicity has recently been evaluated by Pace et al. in a randomized, placebo-controlled trial [52]. This was a phase III study in which 108 patients, treated with cisplatin, were randomized to receive vitamin E (alpha-tocopherol $400 \mathrm{mg} /$ day) or placebo. Class II evidence that vitamin E supplementation significantly reduces the relative risk of developing signs or symptoms of neurotoxicity (relative risk $=0.14)(95 \%$ confidence interval $=0.02-1.00, P<0.05)$ was provided [52].

5.3. Calcium ( $\mathrm{Ca}$ ) and Magnesium ( $\mathrm{Mg}$ ) Infusions. $\mathrm{Ca} / \mathrm{Mg}$ infusions have been used to decrease the incidence of oxaliplatin-induced neuropathy without any influence on antitumor activity [53-55]. However, the CONCEPT study reported that treatment with $\mathrm{Ca} / \mathrm{Mg}$ decrease antitumor effect in patients with metastatic colorectal cancer treated with oxaliplatin, and thus, they are not advisable in combination with the FOLFOX regimen [56].

5.4. Anticonvulsants (Gabapentin and Pregabalin). Gabapentin is an antiepileptic drug which has been used in the management of neuropathic pain. Some studies attempted to assess the impact of gabapentin on oxaliplatin-induced neurotoxicity, but they could not support a role for gabapentin in reducing the severity of oxaliplatin-induced neurotoxicity [57-59].

Pregalin is also an anticonvulsant drug used for neuropathic pain. A case of successful treatment of hyperexcitability syndrome with pregalin after oxaliplatin and gemcitabine therapy for pancreatic cancer has been described [60]. In a recent study, Saif et al. treated 23 patients with gastrointestinal tumors and grade 2 and 3 oxaliplatininduced neurotoxicity with pregabalin at a dose of $150 \mathrm{mg}$ orally 3 times a day and found that pregabalin reduced the severity of oxaliplatin-induced neuropathy [61].

5.5. Other Strategies. Nonpharmacological approaches to prevent oxaliplatin-induced neurotoxicity include the "stop and go" concept. This strategy is based on the observation of reversibility of neurotoxic symptoms after discontinuation of oxaliplatin. In this approach, patients receive treatment with oxaliplatin plus $5 \mathrm{FU} / \mathrm{LV}$ until either the beginning of the development of peripheral neurotoxicity or a predetermined "time to best response". Next, maintain ace therapy with $5 \mathrm{FU} / \mathrm{LV}$ is continued without oxaliplatin so that any neurologic damage is given time to recede. Subsequently, oxaliplatin is reintroduced to maximize the potential effect of the combination regime [62].

Another strategy suggested by Petrioli et al. involves longer duration of oxaliplatin administration which is supposed to result in decreased neurotoxicity [63].

\section{Conclusions}

Platinum compounds are active in the treatment of solid tumors, but peripheral neuropathy is the major nonhematological dose-limiting adverse effect especially for cisplatin and oxaliplatin. Despite the efforts to find specific agents who will prevent or minimize neurotoxicity caused by platinum drugs, there is no effective strategy for the management of the neurotoxicity induced by platinum agents. Unfortunately, none of the symptomatic treatments discussed above have proven useful. Therefore, new drugs or strategies for the prevention and amelioration of platinuminduced neurotoxicity must be found.

\section{References}

[1] A. H. Calvert, S. J. Harland, D. R. Newell et al., "Early clinical studies with cis-diammine-1,1-cyclobutane dicarboxylate platinum II," Cancer Chemotherapy and Pharmacology, vol. 9, no. 3, pp. 140-147, 1982.

[2] R. S. Go and A. A. Adjei, "Review of the comparative pharmacology and clinical activity of cisplatin and carboplatin," Journal of Clinical Oncology, vol. 17, no. 1, pp. 409-422, 1999.

[3] D. M. M. Woloschuk, J. M. Pruemer, and R. J. Cluxton Jr., "Carboplatin: a new cisplatin analog," Drug Intelligence and Clinical Pharmacy, vol. 22, no. 11, pp. 843-849, 1988.

[4] D. J. Higby, H. J. Wallace Jr., D. J. Albert, and J. F. Holland, "Diaminodichloroplatinum: a phase I study showing responses in testicular and other tumors," Cancer, vol. 33, no. 5, pp. 1219-1225, 1974.

[5] J. P. Neijt, S. A. Engelholm, M. K. Tuxen et al., "Exploratory phase III study of paclitaxel and cisplatin versus paclitaxel and carboplatin in advanced ovarian cancer," Journal of Clinical Oncology, vol. 18, no. 17, pp. 3084-3092, 2000. 
[6] M. K. Parmar, J. A. Ledermann, N Colombo et al., "Paclitaxel plus platinum-based chemotherapy versus conventional platinum-based chemotherapy in women with relapsed ovarian cancer: the ICON4/AGO-OVAR-2.2 trial," Lancet, vol. 361, no. 9375, pp. 2099-2106, 2003.

[7] J. S. de Cos Escuín, I. U. Delgado, J. C. Rodríguez, M. J. López, C. D. Vicente, and J. A. Miranda, "Stage IIIA and IIIB nonsmall cell lung cancer: results of chemotherapy combined with radiation therapy and analysis of prognostic factors," Archivos de Bronconeumologia, vol. 43, no. 7, pp. 358-365, 2007.

[8] R. M. Goldberg, D. J. Sargent, R. F. Morton et al., "A randomized controlled trial of fluorouracil plus leucovorin, irinotecan, and oxaliplatin combinations in patients with previously untreated metastatic colorectal cancer," Journal of Clinical Oncology, vol. 22, no. 1, pp. 23-30, 2004.

[9] R. F. Ozols and R. C. Young, "High-dose cisplatin therapy in ovarian cancer," Seminars in Oncology, vol. 12, no. 4, pp. 2130, 1985.

[10] J. S. Gill and A. J. Windebank, "Cisplatin-induced apoptosis in rat dorsal root ganglion neurons is associated with attempted entry into the cell cycle," Journal of Clinical Investigation, vol. 101, no. 12, pp. 2842-2850, 1998.

[11] R. G. van der Hoop, M. E. L. van der Burg, W. W. ten Bokkel Huinink, J. C. van Houwelingen, and J. P. Neijt, "Incidence of neuropathy in 395 patients with ovarian cancer treated with or without cisplatin," Cancer, vol. 66, no. 8, pp. 1697-1702, 1990.

[12] R. J. Cersosimo, "Cisplatin neurotoxicity," Cancer Treatment Reviews, vol. 16, no. 4, pp. 195-211, 1989.

[13] R. W. Gregg, J. M. Molepo, V. J. A. Monpetit et al., "Cisplatin neurotoxicity: the relationship between dosage, time, and platinum concentration in neurologic tissues, and morphologic evidence of toxicity," Journal of Clinical Oncology, vol. 10, no. 5, pp. 795-803, 1992.

[14] S. W. Thompson, L. E. Davis, M. Kornfeld, R. D. Hilgers, and J. C. Standefer, "Cisplatin neuropathy. Clinical, electrophysiologic, morphologic, and toxicologic studies," Cancer, vol. 54, no. 7, pp. 1269-1275, 1984.

[15] R. I. Roelofs, W. Hrushesky, J. Rogin, and L. Rosenberg, "Peripheral sensory neuropathy and cisplatin chemotherapy," Neurology, vol. 34, no. 7, pp. 934-938, 1984.

[16] D. D. Von Hoff, R. Schilsky, C. M. Reichert et al., "Toxic effects of cis-dichlorodiammineplatinum(II) in man," Cancer Treatment Reports, vol. 63, no. 9-10, pp. 1527-1531, 1979.

[17] W. Bruck, E. Heise, and R. L. Friede, "Leukoencephalopathy after cisplatin therapy," Clinical Neuropathology, vol. 8, no. 6, pp. 263-265, 1989.

[18] M. T. Cattaneo, V. Filipazzi, E. Piazza, E. Damiani, and G. Mancarella, "Transient blindness and seizure associated with cisplatin therapy," Journal of Cancer Research and Clinical Oncology, vol. 114, no. 5, pp. 528-530, 1988.

[19] M. von Schlippe, C. J. Fowler, and S. J. Harland, "Cisplatin neurotoxicity in the treatment of metastatic germ cell tumour: time course and prognosis," British Journal of Cancer, vol. 85, no. 6, pp. 823-826, 2001.

[20] J. T. Hartmann and H. P. Lipp, "Toxicity of platinum compounds," Expert Opinion on Pharmacotherapy, vol. 4, no. 6, pp. 889-901, 2003.

[21] G. Laurell, C. Beskow, B. Frankendal, and E. Borg, "Cisplatin administration to gynecologic cancer patients: long term effects on hearing," Cancer, vol. 78, no. 8, pp. 1798-1804, 1996.

[22] D. Glover, J. H. Glick, C. Weiler, J. Yuhas, and M. M. Kligerman, "Phase I trials of WR-2721 and cis-platinum," International Journal of Radiation Oncology Biology Physics, vol. 10, pp. 1781-1784, 1984.
[23] R. J. Hallmark, J. M. Snyder, K. Jusenius, and H. K. Tamimi, "Factors influencing ototoxicity in ovarian cancer patients treated with cis-platinum based chemotherapy," European Journal of Gynaecological Oncology, vol. 13, no. 1, pp. 35-44, 1992.

[24] U. Peters, S. Preisler-Adams, A. Hebeisen et al., "Glutathione S-transferase genetic polymorphisms and individual sensitivity to the ototoxic effect of cisplatin," Anti-Cancer Drugs, vol. 11, no. 8, pp. 639-643, 2000.

[25] M. J. Moroso and R. L. Blair, "A review of cis-platinum ototoxicity," Journal of Otolaryngology, vol. 12, no. 6, pp. 365369, 1983.

[26] P. Chapman, "Rapid onset hearing loss after cisplatinum therapy: case reports and literature review," Journal of Laryngology and Otology, vol. 96, no. 2, pp. 159-162, 1982.

[27] M. J. McKeage, "Comparative adverse effect profiles of platinum drugs," Drug Safety, vol. 13, no. 4, pp. 228-244, 1995.

[28] R. Canetta, M. Rozencweig, and S. K. Carter, "Carboplatin: the clinical spectrum to date," Cancer Treatment Reviews, vol. 12, supplement, pp. 125-136, 1985.

[29] S. R. McWhinney, R. M. Goldberg, and H. L. McLeod, "Platinum neurotoxicity pharmacogenetics," Molecular Cancer Therapeutics, vol. 8, no. 1, pp. 10-16, 2009.

[30] O. Heinzlef, J. P. Lotz, and E. Roullet, "Severe neuropathy after high dose carboplatin in three patients receiving multidrug chemotherapy," Journal of Neurology Neurosurgery and Psychiatry, vol. 64, no. 5, pp. 667-669, 1998.

[31] E. Cvitkovic, "Cumulative toxicities from cisplatin therapy and current cytoprotective measures," Cancer Treatment Reviews, vol. 24, no. 4, pp. 265-281, 1998.

[32] H. Adelsberger, S. Quasthoff, J. Grosskreutz, A. Lepier, F. Eckel, and C. Lersch, "The chemotherapeutic oxaliplatin alters voltage-gated $\mathrm{Na}^{+}$channel kinetics on rat sensory neurons," European Journal of Pharmacology, vol. 406, no. 1, pp. 25-32, 2000.

[33] E. Gamelin, L. Gamelin, L. Bossi, and S. Quasthoff, "Clinical aspects and molecular basis of oxaliplatin neurotoxicity: current management and development of preventive measures," Seminars in Oncology, vol. 29, no. 5, pp. 21-33, 2002.

[34] J. Cassidy and J. L. Misset, "Oxaliplatin-related side effects: characteristics and management," Seminars in Oncology, vol. 29, no. 5, pp. 11-20, 2002.

[35] P. C. Kurniali, L. G. Luo, and A. B. Weitberg, "Role of calcium/ magnesium infusion in oxaliplatin-based chemotherapy for colorectal cancer patients," Oncology, vol. 24, no. 3, pp. 289292, 2010.

[36] A. A. Argyriou, P. Polychronopoulos, G. Iconomou, E. Chroni, and H. P. Kalofonos, "A review on oxaliplatin-induced peripheral nerve damage," Cancer Treatment Reviews, vol. 34, no. 4, pp. 368-377, 2008.

[37] G. Cavaletti and C. Zanna, "Current status and future prospects for the treatment of chemotherapy-induced peripheral neurotoxicity," European Journal of Cancer, vol. 38, no. 14, pp. 1832-1837, 2002.

[38] S. Taieb, V. Trillet-Lenoir, L. Rambaud, L. Descos, and G. Freyer, "Lhermitte sign and urinary retention: atypical presentation of oxaliplatin neurotoxicity in four patients," Cancer, vol. 94, no. 9, pp. 2434-2440, 2002.

[39] A. Grothey, "Clinical management of oxaliplatin-associated neurotoxicity," Clinical Colorectal Cancer, vol. 5, supplement 1, pp. S38-S46, 2005.

[40] J. M. Extra, M. Marty, S. Brienza, and J. L. Misset, "Pharmacokinetics and safety profile of oxaliplatin," Seminars in Oncology, vol. 25, no. 2, pp. 13-22, 1998. 
[41] V. Hellberg, I. Wallin, S. Eriksson et al., "Cisplatin and oxaliplatin toxicity: importance of cochlear kinetics as a determinant for ototoxicity," Journal of the National Cancer Institute, vol. 101, no. 1, pp. 37-47, 2009.

[42] N. K. Malhotra, R. Aslam, S. P. Lipman, and V. J. Bilski, "Acute ototoxicity from a single infusion of oxaliplatin," Ear, Nose and Throat Journal, vol. 89, no. 6, pp. 258-261, 2010.

[43] G. Kemp, P. Rose, J. Lurain et al., "Amifostine pretreatment for protection against cyclophosphamide-induced and cisplatininduced toxicities: results of a randomized control trial in patients with advanced ovarian cancer," Journal of Clinical Oncology, vol. 14, no. 7, pp. 2101-2112, 1996.

[44] D. Screnci and M. J. McKeage, "Platinum neurotoxicity: clinical profiles, experimental models and neuroprotective approaches," Journal of Inorganic Biochemistry, vol. 77, no. 1-2, pp. 105-110, 1999.

[45] S. Cascinu, L. Cordella, E. Del Ferro, M. Fronzoni, and G. Catalano, "Neuroprotective effect of reduced glutathione on cisplatin-based chemotherapy in advanced gastric cancer: a randomized double-blind placebo- controlled trial," Journal of Clinical Oncology, vol. 13, no. 1, pp. 26-32, 1995.

[46] J. F. Smyth, A. Bowman, T. Perren et al., "Glutathione reduces the toxicity and improves quality of life of women diagnosed with ovarian cancer treated with cisplatin: results of a doubleblind, randomised trial," Annals of Oncology, vol. 8, pp. 569573, 1997.

[47] S. Cascinu, V. Catalano, L. Cordella et al., "Neuroprotective effect of reduced glutathione on oxaliplatin-based chemotherapy in advanced colorectal cancer: a randomized, doubleblind, placebo-controlled trial," Journal of Clinical Oncology, vol. 20, no. 16, pp. 3478-3483, 2002.

[48] M. Dong, P. Y. Xing, P. Liu, F. Y. Feng, and Y. K. Shi, "Assessment of the protective effect of calcium-magnesium infusion and glutathione on oxaliplatin-induced neurotocixity," Chinese Journal of Oncology, vol. 32, pp. 208-211, 2010.

[49] B. A. Arrick and C. F. Nathan, "Glutathione metabolism as a determinant of therapeutic efficacy: a review," Cancer Research, vol. 44, no. 10, pp. 4224-4232, 1984.

[50] S. E. Bates, J. I. Regis, R. W. Robey, Z. Zhan, S. Scala, and B. J. Meadows, "Chemoresistance in the clinic: overview 1994," Bulletin du Cancer, vol. 81, supplement 2, pp. 55-61, 1994.

[51] J. A. Foster-Nora and R. Siden, "Amifostine for protection from antineoplastic drug toxicity," American Journal of HealthSystem Pharmacy, vol. 54, no. 7, pp. 787-800, 1997.

[52] A. Pace, D. Giannarelli, E. Galiè et al., "Vitamin E neuroprotection for cisplatin neuropathy: a randomized, placebocontrolled trial," Neurology, vol. 74, no. 9, pp. 762-766, 2010.

[53] M. W. Saif, "Oral calcium ameliorating oxaliplatin-induced peripheral neuropathy," Journal of Applied Research, vol. 4, no. 4, pp. 576-582, 2004.

[54] K. Ishibashi, N. Okada, T. Miyazaki, M. Sano, and H. Ishida, "Effect of calcium and magnesium on neurotoxicity and blood platinum concentrations in patients receiving mFOLFOX6 therapy: a prospective randomized study," International Journal of Clinical Oncology, vol. 15, no. 1, pp. 82-87, 2010.

[55] L. Gamelin, M. Boisdron-Celle, R. Delva et al., "Prevention of oxaliplatin-related neurotoxicity by calcium and magnesium infusions: a retrospective study of 161 patients receiving oxaliplatin combined with 5-fluorouracil and leucovorin for advanced colorectal cancer," Clinical Cancer Research, vol. 10, no. 12, pp. 4055-4061, 2004.
[56] H. S. Hochster, A. Grothey, and B. H. Childs, "Use of calcium and magnesium salts to reduce oxaliplatin-related neurotoxicity," Journal of Clinical Oncology, vol. 25, no. 25, pp. 4028-4029, 2007.

[57] P. L. Mitchell, D. Goldstein, M. Michael et al., "Addition of gabapentin to a modified FOLFOX regimen does not reduce oxaliplatin-induced neurotoxicity," Clinical Colorectal Cancer, vol. 6, no. 2, pp. 146-151, 2006.

[58] R. D. Rao, J. C. Michalak, J. A. Sloan et al., "Efficacy of gabapentin in the management of chemotherapy-induced peripheral neuropathy: a phase 3 randomized, double-blind, placebo-controlled, crossover trial (N00C3)," Cancer, vol. 110, no. 9, pp. 2110-2118, 2007.

[59] N. Tsavaris, P. Kopterides, C. Kosmas et al., "Gabapentin monotherapy for the treatment of chemotherapy-induced neuropathic pain: a pilot study," Pain Medicine, vol. 9, no. 8, pp. 1209-1216, 2008.

[60] M. W. Saif and S. Hashmi, "Successful amelioration of oxaliplatin-induced hyperexcitability syndrome with the antiepileptic pregabalin in a patient with pancreatic cancer," Cancer Chemotherapy and Pharmacology, vol. 61, no. 3, pp. 349-354, 2008.

[61] M. W. Saif, K. Syrigos, K. Kaley, and I. Isufi, "Role of pregabalin in treatment of oxaliplatin-induced sensory neuropathy," Anticancer Research, vol. 30, no. 7, pp. 2927-2933, 2010.

[62] C. Tournigand, A. Cervantes, A. Figer et al., "OPTIMOX1: a randomized study of FOLFOX4 or FOLFOX7 with oxaliplatin in a stop-and-go fashion in advanced colorectal cancer-a GERCOR study," Journal of Clinical Oncology, vol. 24, no. 3, pp. 394-400, 2006.

[63] R. Petrioli, A. Pascucci, E. Francini et al., "Neurotoxicity of FOLFOX-4 as adjuvant treatment for patients with colon and gastric cancer: a randomized study of two different schedules of oxaliplatin," Cancer Chemotherapy and Pharmacology, vol. 61, no. 1, pp. 105-111, 2008. 


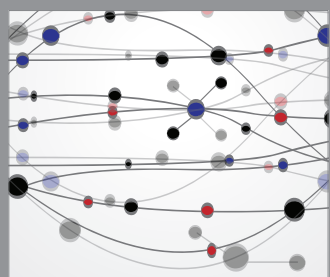

The Scientific World Journal
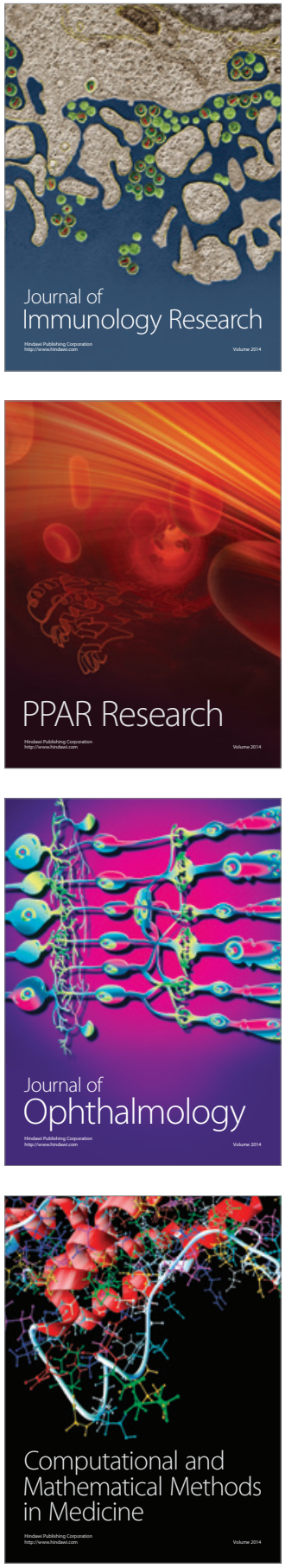

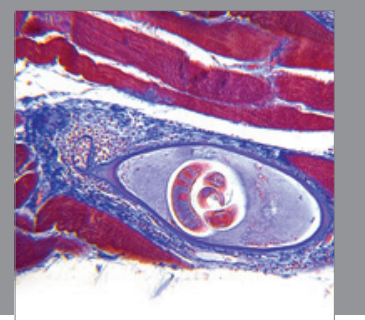

Gastroenterology

Research and Practice
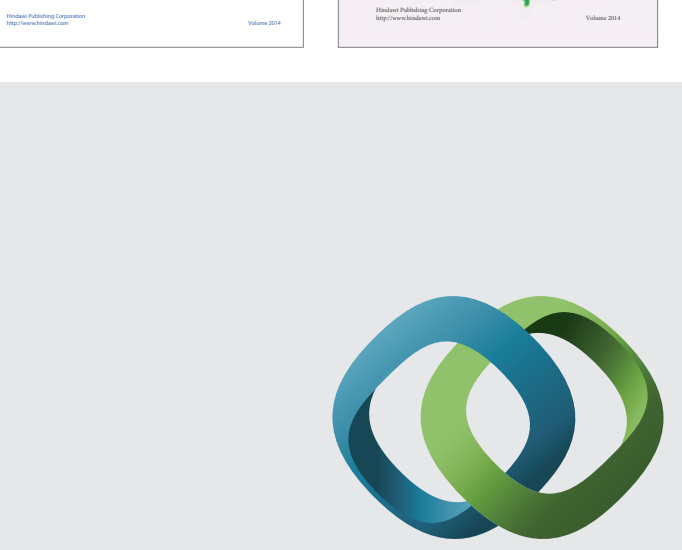

\section{Hindawi}

Submit your manuscripts at

http://www.hindawi.com
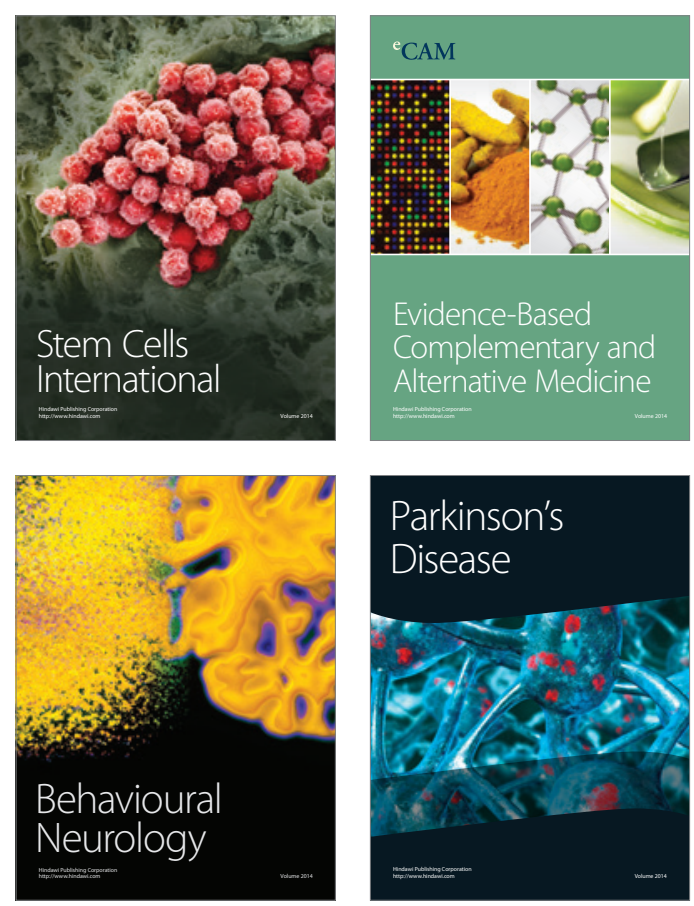

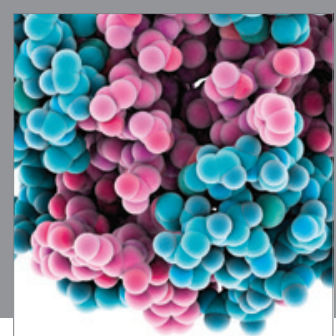

Journal of
Diabetes Research

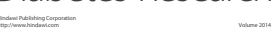

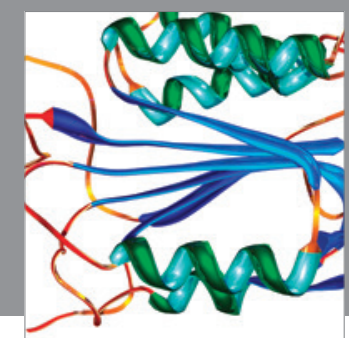

Disease Markers
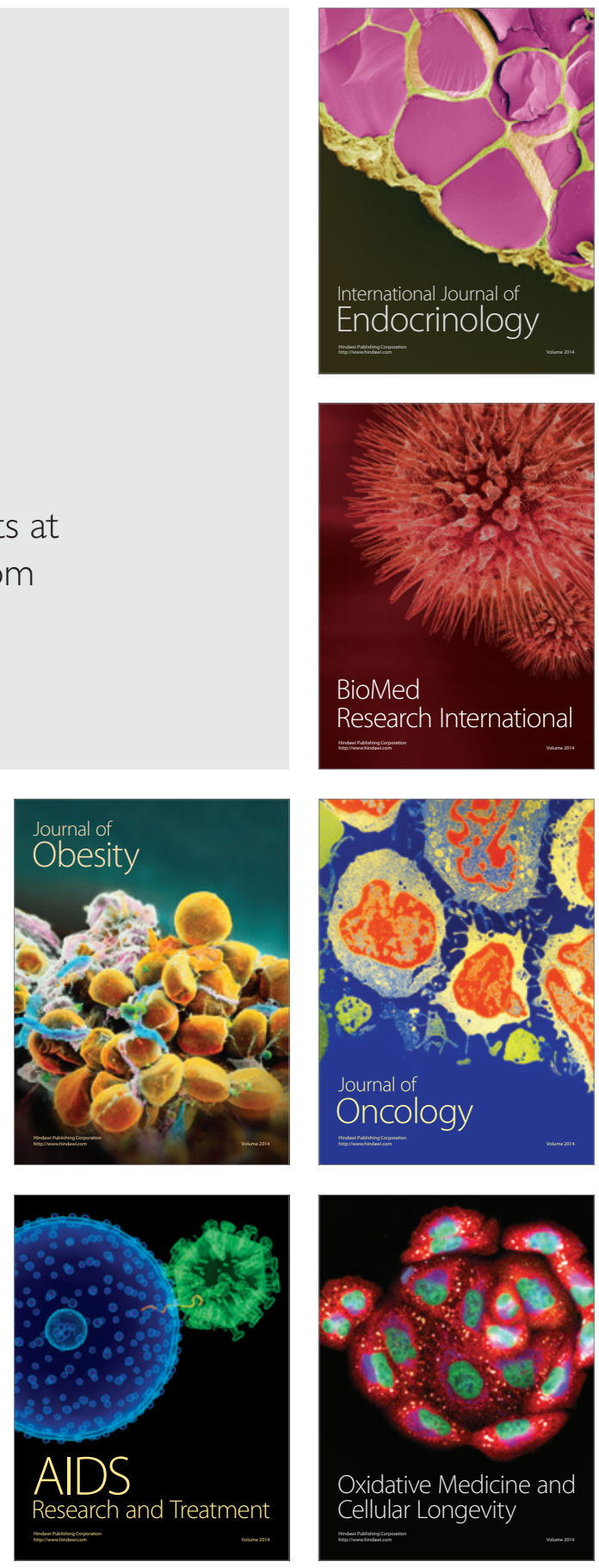